\title{
Distinct cortical networks for the detection and identification of human body
}

Citation for published version (APA):

Hodzic, A., Kaas, A. L., Muckli, L., Stirn, A., \& Singer, W. (2009). Distinct cortical networks for the detection and identification of human body. Neuroimage, 45(4), 1264-1271.

https://doi.org/10.1016/j.neuroimage.2009.01.027

Document status and date:

Published: 01/01/2009

DOI:

10.1016/j.neuroimage.2009.01.027

Document Version:

Publisher's PDF, also known as Version of record

Document license:

Taverne

Please check the document version of this publication:

- A submitted manuscript is the version of the article upon submission and before peer-review. There can be important differences between the submitted version and the official published version of record.

People interested in the research are advised to contact the author for the final version of the publication, or visit the DOI to the publisher's website.

- The final author version and the galley proof are versions of the publication after peer review.

- The final published version features the final layout of the paper including the volume, issue and page numbers.

Link to publication

\footnotetext{
General rights rights.

- You may freely distribute the URL identifying the publication in the public portal. please follow below link for the End User Agreement:

www.umlib.nl/taverne-license

Take down policy

If you believe that this document breaches copyright please contact us at:

repository@maastrichtuniversity.nl

providing details and we will investigate your claim.
}

Copyright and moral rights for the publications made accessible in the public portal are retained by the authors and/or other copyright owners and it is a condition of accessing publications that users recognise and abide by the legal requirements associated with these

- Users may download and print one copy of any publication from the public portal for the purpose of private study or research.

- You may not further distribute the material or use it for any profit-making activity or commercial gain

If the publication is distributed under the terms of Article $25 \mathrm{fa}$ of the Dutch Copyright Act, indicated by the "Taverne" license above, 


\title{
Distinct cortical networks for the detection and identification of human body
}

\author{
Amra Hodzic a,b,c,*, Amanda Kaas a,b,d, Lars Muckli a,b,e , Aglaja Stirn ${ }^{\text {a,f }}$, Wolf Singer ${ }^{\text {a,b }}$ \\ a Department of Neurophysiology, Max Planck Institute for Brain Research, Deutschordenstr. 46, D-60528 Frankfurt am Main, Germany \\ ${ }^{\mathrm{b}}$ Brain Imaging Center Frankfurt, Schleusenweg 2-16, D-60528 Frankfurt am Main, Germany \\ c Graduate School of Neural and Behavioural Sciences, IMPRS, Österbergstraße 3, D-72074 Tübingen, Germany \\ d Department of Cognitive Neuroscience, Faculty of Psychology, Maastricht University, Postbus 616, 6200 MD Maastricht, The Netherlands \\ e Department of Psychology and Center for Cognitive Neuroimaging CCNi, 58 Hillhead Street, University of Glasgow, Glasgow G12 8QB, UK \\ ${ }^{\mathrm{f}}$ Department of Psychiatry, University Hospital Frankfurt, Heinrich-Hoffmann-Straße 10, D-60528 Frankfurt am Main, Germany
}

\section{A R T I C L E I N F O}

\section{Article history:}

Received 14 August 2008

Revised 15 January 2009

Accepted 15 January 2009

Available online 6 February 2009

\begin{abstract}
A B S T R A C T
In the human brain information about bodies and faces is processed in specialized cortical regions named EBA and FBA (extrastriate and fusiform body area) and OFA and FFA (occipital and fusiform face area), respectively. Here we investigate with functional magnetic resonance imaging (fMRI) the cortical areas responsible for the identification of individual bodies and the distinction between 'self and 'others'. To this end we presented subjects with images of unfamiliar and familiar bodies and their own body. We identified separate coactivation networks for body-detection (processing body related information), body-identification (processing of information relating to individual bodies) and self-identification (distinction of self from others). Body detection involves the EBA in both hemispheres, and in the right hemisphere: the FBA and areas in the IPL (inferior parietal lobe). Body identification involves areas in the inferior frontal gyrus (IFG) of both hemispheres and in the right hemisphere areas in the medial frontal gyrus (MFG), in the cingulate gyrus (CG), in the central (CS) and the post-central sulcus (PCS), in the inferior parietal lobe (IPL) and the FBA When the recognition of one's own body is contrasted to the identification of familiar bodies, differential activation is observed in areas of the inferior parietal lobe (IPL) and inferior parietal sulcus (IPS) of the right hemisphere, and in the posterior orbital gyrus (pOrbG) and in the lateral occipital gyrus (LOG) of the left hemisphere. Thus, identification of individual bodies and self-other distinction involve in addition to the classical occipito-parietal network a parieto-frontal network. Interestingly, the EBA shows no differential activation for distinctions between familiar or unfamiliar bodies or recognition of one's own body.
\end{abstract}

(C) 2009 Elsevier Inc. All rights reserved.

\section{Introduction}

Identification and recognition of individuals rely to a large extent on visual information about features of faces and bodies and so do judgments on the emotional state and intentions of the respective others. Accordingly, it is argued that there are areas of the cerebral cortex specialized for the processing of body and face related information. These are the EBA and FBA (extrastriate and fusiform body area) and OFA and FFA (occipital and fusiform face area), respectively (Puce et al., 1996, Kanwisher et al., 1997, Downing et al., 2001, Peelen and Downing 2005, Schwarzlose et al., 2005, Downing et al., 2006, Peelen et al., 2006). These are the EBA and FBA (extrastriate and fusiform body area) and OFA and FFA (occipital and fusiform face area), respectively (Puce et al., 1996, Kanwisher et al., 1997, Downing et al., 2001, Peelen and Downing 2005, Schwarzlose et al., 2005, Downing et al., 2006, Peelen et al., 2006).

\footnotetext{
* Corresponding author. Department of Neurophysiology, Max Planck Institute for Brain Research, Deutschordenstr. 46, D-60528 Frankfurt am Main, Germany. Fax: +4969 96769327.

E-mail address: hodzic@mpih-frankfurt.mpg.de (A. Hodzic).
}

The first localizations of areas in the human visual cortex responding selectively to images of bodies and body parts have been performed with functional Magnetic Resonance Imaging (fMRI) in humans (Downing et al., 2001) and in monkeys (Pinsk et al., 2005, Tsao et al., 2003), and confirmed with additional methods such as Evoked Potentials (EP) (Kovacs et al., 2005, Thierry et al., 2006), Intracranial Recordings (Pourtois et al., 2007) and Transcranial Magnetic Stimulation (TMS) (Urgesi et al., 2004, Urgesi et al., 2007a,b). Single-cell recordings in non-human primates also point to a remarkable functional selectivity of neurons for shapes of bodies and body parts in the inferior temporal cortex (Gross et al., 1972, DeSimone et al., 1984, Wachsmuth et al., 1994, Tsao et al., 2006, Kiani et al., 2007).

However, the notion of distinct, category specific cortical regions is still a matter of debate. There is also evidence that the representations of faces and objects in ventral temporal cortex are widely distributed and overlapping (Haxby et al., 2001, Ishai et al., 2000, O'Toole et al., 2005) and that the specialization of the face selective area is dependent on expertise (Gauthier et al., 1999). Thus, local specialization and distributedness coexist and further investigations are needed to unveil the nature of cortical representations. 
Faces and bodies constitute a unique object category because they can be further classified according to identity and ownership. Together with gait, gesture and prosody faces and bodies are crucial for the assessment of a person's identity. Visual information extractable from faces and bodies allows for judgments on gender, age, and ethnicity and ultimately the identity of a person. This includes as special case the identification of a body or a face as belonging to oneself or someone else.

The distinction between self and other and the representation of the self rely on numerous sources of information. Proprioceptive and visual signals contribute to the representation of the dynamic body scheme and the awareness of ownership of the body (Jackson and Decety 2004, Uher et al., 2005, Pellijeff et al., 2006, Shimada et al., 2005, Vogeley et al., 2001). Furthermore, investigations of the sense of agency revealed differential cortical activation patterns for the anticipation, visualization, and observation of one's own movement versus that of others (Chaminade and Decety 2002, Cunnington et al., 2006, Grezes et al., 2004, Ramnani and Miall 2004). Because of the prominent role of facial cues for the discrimination of an person's identity the studies on self-representation and self vs. others distinction are based on the distinction between one's own face and that of others (Platek et al., 2006, Uddin et al., 2005, 2006). In the present study we investigated networks involved in self-other distinction that rely on non-facial cues. To this end we presented images of headless bodies and examined whether self-body-identification is reflected in the differential activation of the cortical areas involved in the encoding of bodies or body parts (EBA and FBA) or whether it involves in addition specific networks devoted to the distinction between self and other in a more general sense.

We examined subjects with event related fMRI while they performed two explicit two-alternative, forced-choice identification tasks: 1. distinction of one's own body from other unfamiliar bodies and 2. distinction of a familiar body from other unfamiliar bodies. This paradigm was used in order to dissociate 'familiarity' from the distinction between 'self' and 'other'. In a previous study, based on a block design and passive viewing conditions, we found that cortical areas responding to bodies and self-other distinctions were not restricted to the extrastriate visual cortex (Hodzic et al., 2008). In the current study, we applied a block design to identify body related areas and an event related design for the segregation of areas involved in the self-other distinction, manipulating the identity, familiarity and the viewpoint of the presented body images.

\section{Methods}

Ten human volunteers (mean age 27 years, 5 females) with normal or corrected-to-normal vision took part in this study. All participants gave their informed written consent to the procedure in accordance with institutional guidelines and the Helsinki declaration (www.wma.net/e/ethicsunit/helsinki.htm).

For the compilation of the stimulus material subjects wearing dark blue bikinis or swimming suits were photographed in neutral, frontal, upright posture against a non-reflecting gray background. The photographs were taken with a digital camera Canon EOS-30D, (3.1 million effective pixels) and edited with PHOTO-PAINT X3 graphic suite in order to obtain gray-scale images of headless bodies against a uniform gray background $(400 \times 400)$. A mirrored version was also produced for each body picture.

In the scanner stimuli were back-projected onto a frosted screen with a liquid-crystal-display projector (VPL PX 20, Sony, Tokyo, Japan) and a custom-made lens. Subjects were viewing the screen through a mirror fixed onto the head coil. Stimulus sequences were generated with Presentation 9.90 software (http://nbs.neuro-bs. com). We performed two types of fMRI experiments, in the first, we used a block design to map body selective areas, in the second, we applied a rapid event related design, to assess cortical activity related to body identification and self versus other distinctions.

Even though the localization of functional regions of interest (fROIs) can lead to a biased and inappropriately constrained characterization of functional anatomy (Friston et al., 2006), we used this approach for two reasons. First, this procedure identifies fROIs that are anatomically more consistent and second, it constrains the number of fROIs, and thereby increases statistical power (Saxe et al., 2006a,b).

\section{Mapping of body selective regions}

Each subject took part in two runs of the mapping experiment. Subjects were asked to fixate a cross that was continuously present in the center of the screen. Stimuli consisted of blocks of images of bodies, images of neutral objects, and blanks with only the fixation cross present. In each run, the first block was repeated five times and the other four times, compensating for the fact that the initial four scan volumes were discarded to avoid T1 saturation effects. One run lasted 4.43 min during which 266 volumes were recorded. Each block lasted $16 \mathrm{~s}$ and comprised 16 volumes. Within a block, 16 pictures of one category were presented. One picture was shown for $600 \mathrm{~ms}$ followed by a $400 \mathrm{~ms}$ blank interval. The pictures were randomized within and between blocks, and the blocks' order was counterbalanced within and between subjects using MATLAB (6.5). In order to maintain a constant level of vigilance, subjects performed a 'one back repetition task', indicating the repetition of an image by pressing a button.

\section{Body identification tasks}

To examine cortical activity related to the distinction between self and other, subjects were asked to recognize their own body, the bodies of unknown others and the bodies of familiar other. The concept of 'familiarity' was introduced to separate identification of familiar bodies from the identification of the own body.

For familiarization we selected two pictures from our databank and named them 'Elli' (female body) and 'Egon' (male body). Each subject was familiarized with another body of the same sex. The subjects could take as much time as they needed (average $4 \mathrm{~min}$ ) to familiarize themselves with the body images (camera and mirror version) on a computer monitor before going into the scanner. In the scanner, and prior to the measurements we confirmed that subjects were able to recognize 'Egon' or 'Elli' in images presented four times for $1 \mathrm{~s}$.

For the 'unknown other' category two images of unknown body (each from 2 perspectives) were chosen, the images differed across subjects and runs. Four experimental runs ( $2 \times$ 'self' vs. 'unknown other' distinction, $2 \times$ 'familiar other' vs. 'unknown other' distinction) were performed in randomized order across and within subjects. Subjects were asked to respond by a button press with the right or left hand when they recognized shown body as being familiar ('self' or 'familiar other') or unfamiliar ('unknown other'). Assignment of the left and right hand was randomized across subjects. Subjects were instructed prior to each run whether they would have to perform the 'self vs. 'unknown other' or the 'familiar other' vs. 'unknown other' distinction. Overall, the images of 'familiar other', 'self' and two sets of 'unfamiliar body' were presented 46 times (23 times per perspective) during the experiment. The reaction times and the accuracy of each response were recorded.

We used a rapid event related design where each run consisted of 46 trials per condition (including fixation). The conditions were: fixation, 'unknown other body', 'self and 'familiar other'. Within each condition (except fixation) equal numbers of mirror and camera images were presented. One trial lasted $3000 \mathrm{~ms}$ with $800 \mathrm{~ms}$ of image presentation and $2200 \mathrm{~ms}$ of interstimulus interval (only fixation cross). Each run began and ended with a fixation period of $6000 \mathrm{~ms}$. In sum, one run consisted of 184 trials or 552 
fMRI volumes (9.2 min). Trial order was randomized across conditions in such a way that trials from each condition (fixation included) were preceded equally often by trials from all four conditions (two-back randomization).

\section{fMRI measurement}

Scanning was performed with a 3T MR scanner (Siemens Allegra, Erlangen, Germany). A gradient-recalled echo-planar-imaging sequence (EPI) was applied with the following parameters: 18 slices oriented approximately parallel to the anterior-posterior commissure plane; TR $1000 \mathrm{~ms}$; TE $30 \mathrm{~ms}$; FA 77; FOV $210 \mathrm{~mm}$; in-plane resolution $3.14 \times 3.14 \mathrm{~mm}$; slice thickness $5 \mathrm{~mm}$ and gap size $0.5 \mathrm{~mm}$. In addition, a T1-weighted anatomical scan was acquired with a magnetization-prepared rapid-acquisition gradient-echo (MPRAGE). In all subjects, functional EPI scans were automatically corrected for geometric distortions by the reconstruction PSF (Point Spread Function) algorithm. PSF is an algorithm that enables reliable and fully automated distortion correction of EPI scans at high field strength (Zaitsev et al., 2004).

\section{Data analysis}

Data analysis and visualization were performed using the BrainVoyager QX software package (Brain Innovation, Maastricht, The Netherlands). The first four volumes of each run were discarded to preclude T1 saturation effects. Pre-processing of the functional data included motion correction, linear trend removal and temporal highpass filtering at $0.01 \mathrm{~Hz}$, slice-scan-time correction and spatial smoothing using an $8 \mathrm{~mm}$ kernel. For each subject the functional and structural 3D data sets were transformed into Talairach coordinate space (Talairach and Tournoux, 1988).

For the mapping experiment statistical examination was based on the general linear model (GLM) employing a boxcar function for the stimulation and fixation conditions, convolved with a gamma function to account for the shape and delay of the hemodynamic response. Initially, we performed an explorative whole brain single-subject analysis to identify body selective regions in the left and right hemisphere. Body selective ROIs were defined in each individual by a whole brain GLM contrast for bodies versus objects (bodies $>$ objects). ROIs were selected as clusters of voxels ( $>20$ neighbouring voxels) that exceeded the specified threshold. In order to match the number of selected voxels per ROI across subjects the thresholds were adjusted for each subject individually, ranging between $t>1.76$ and $t>6.64$, or $p<0.01$ to $p<0.000$ (see the Table 1 for exact values). Subsequently, we carried out explorative whole brain multi-subject analysis for the contrast bodies $>$ objects after individual Talairach transformation. The contrast was calculated using GLM random effect (RFX) analyses at the threshold $t>3$ and $p<0.05$ corrected for multiple comparisons. As a result we defined specific cortical areas involved in general processing of body related information irrespective of identity or familiarity. Thus, we named these body-processing areas the "body-detection network". We used the term 'network' to highlight that not a single cortical area but several distant regions are responding to the same contrast. We did not attempt to make any claim whether these areas are directly or indirectly interconnected with one another at the functional or anatomical level.

The rapid event-related identification experiments used closely spaced trials, leading to a substantial overlap in the resulting hemodynamic responses. Nevertheless, with a balanced randomization (two-back randomization, see above), the underlying hemodynamic responses can be assessed by deconvolution (Dale and Buckner 1997). A deconvolution analysis estimates the hemodynamic response function for each trial on the basis of a general linear model (GLM). Twenty predictors ( $20 \mathrm{~s}$ or $20 \mathrm{fMRI}$ volumes) were defined to cover the temporal extent of a typical hemodynamic response.

We conducted a whole brain multi-subject RFX GLM analysis $(t(9)>3 ; p<0.05$ uncorrected) to investigate which brain regions were influenced by the perspective manipulation (camera or mirror image) using the contrast ([mirror $>$ camera] and [camera $>$ mirror]). Similarly, using RFX GLM analysis we assessed potential effects of a different "task set" in the 'self vs. 'unknown other' and 'familiar other' vs. 'unknown other' runs. Here we compared the response to the unknown other body during different runs ([other 1 ('self run)> other 2 ('familiar other' run)] and ([other $2>$ other 1 ].

In addition, in order to segregate cortical responses devoted to the identification of bodies we conducted a whole brain multisubject FE GLM conjunction analysis for the contrast ([self $>$ others] and [familiar other $>$ others]). The corresponding map was Bonferoni corrected at the $p<0.05$ criterion. Examining responses to these contrasts we could identify the areas that encode information related to individual bodies, the 'body-identification network'. Here we refer to 'network' as described above. Subsequently, we performed multisubject random effect (RFX) GLM analysis to examine contrast [self $>$ familiar other] and define brain regions involved in the distinction of self from others, the 'self-identification network'. The self $>$ familiar other cortical map was corrected for multiple comparisons using the "Cluster threshold estimator plug-in" (BV QX, F. d' Esposito), setting a minimum cluster size corresponding to the 0.05 threshold in a permutation test based null distribution of cluster sizes (Forman et al., 1995). This procedure resulted in a spatial extent threshold of 104 contiguous functional voxels.

Finally, we conducted a multi-subject ROI based analysis of the fROIs of the body detection network. We tested all contrasts of interest ([self $>$ others], [familiar other $>$ others] and [self $>$ familiar others]). Also, we tested whether the pattern of activation for the conditions differed across the fROIs, performing a 4 by 3 repeated measures ANOVA with ROI (right EBA, right FBA, left EBA and right PL) and Condition ('other', 'familiar other', and 'self') as within-subjects factors. For the comparison we took the sum of 5 beta weights around the peak of the deconvolution time course for each condition and individual subject.

We assessed reaction time and accuracy of the subjects' responses in the body identification tasks. The mean reaction times and percentage of correct responses across categories ('self', 'familiar other', other_1 (other in 'self run) and other_2 (other in 'familiar other' run)), perspective (camera or mirror image) and the different body identification tasks ('self' vs 'unknown other' and 'familiar other' vs 'unknown other') were calculated and compared using repeated measure ANOVA statistical tests. The reaction times and accuracy of

Table 1

Responses to multi-subject GLM analysis for the body selective ROIs revealed after the whole brain analysis at: $t(9)>3 ; p<0.05$ (corrected for multiple comparisons)

\begin{tabular}{|c|c|c|c|c|c|c|c|c|c|c|c|c|}
\hline \multirow[t]{3}{*}{ ROI } & \multicolumn{12}{|c|}{ Contrasts } \\
\hline & \multicolumn{3}{|c|}{ Bodies $>$ objects } & \multicolumn{3}{|c|}{ Familiar $>$ others } & \multicolumn{3}{|c|}{ Self $>$ others } & \multicolumn{3}{|c|}{ Self $>$ familiar } \\
\hline & $t$ & se & $p$ & $\bar{t}$ & se & $p$ & $\bar{t}$ & se & $p$ & $\bar{t}$ & se & $p$ \\
\hline$\overline{\text { FBA_RH }}$ & $>2.71$ & 0.09 & $<0.006$ & $>2.99$ & 0.20 & $<0.003$ & $>3.99$ & 0.21 & $<0.000$ & $>-0.93$ & 0.25 & $<0.35$ \\
\hline EBA_RH & $>5.93$ & 0.08 & $<0.000$ & $>0.43$ & 0.21 & $<0.67$ & $>1.46$ & 0.28 & $<0.16$ & $>0.21$ & 0.26 & $<0.83$ \\
\hline IPL_RH & $>3.35$ & 0.07 & $<0.000$ & $>3.51$ & 0.19 & $<0.000$ & $>6.17$ & 0.20 & $<0.000$ & $>1.45$ & 0.25 & $<0.02$ \\
\hline EBA_LH & $>4.27$ & 0.07 & $<0.000$ & $>0.94$ & 0.20 & $<0.34$ & $>3.15$ & 0.20 & $<0.001$ & $>0.33$ & 0.25 & $<0.74$ \\
\hline
\end{tabular}


responses to the 'others 1' and 'others 2' categories were included as separate categories to evaluate the effect of 'task set', since the two body identification tasks were performed in different runs.

\section{Results}

\section{Behavioural measures}

The reaction times (RT) and accuracy of the responses during the identification experiments were as follows: condition 'self'; RT=1027 ms, 98\% correct, condition 'familiar other'; RT=1029 ms, 95\% correct, condition 'other 1' (other in 'self' run); RT =1032 ms, 98\% correct, and condition 'other 2' (other in 'familiar other' run); $\mathrm{RT}=1030 \mathrm{~ms}$, 99\% correct (Fig. 1).

Repeated measures ANOVA revealed that the accuracy for the condition 'familiar other' was significantly lower than for the condition 'other 1' and 'other 2' ( $p<0.005$, Bonferroni corrected), while the accuracy for 'self' was significantly higher than that for 'familiar other' $(p<0.02$ Bonferroni corrected). There was no significant difference between other_1 and other_2 ( $p<0.05$ Bonferroni corrected).

Subsequently we split conditions 'self' and 'familiar other' into mirror and camera categories. The corresponding values were: mirror 'self', RT=1026 ms, 97\% correct; camera 'self', RT=1026 ms, 98\% correct; mirror 'familiar other', RT=1026 ms, 93\% correct and camera
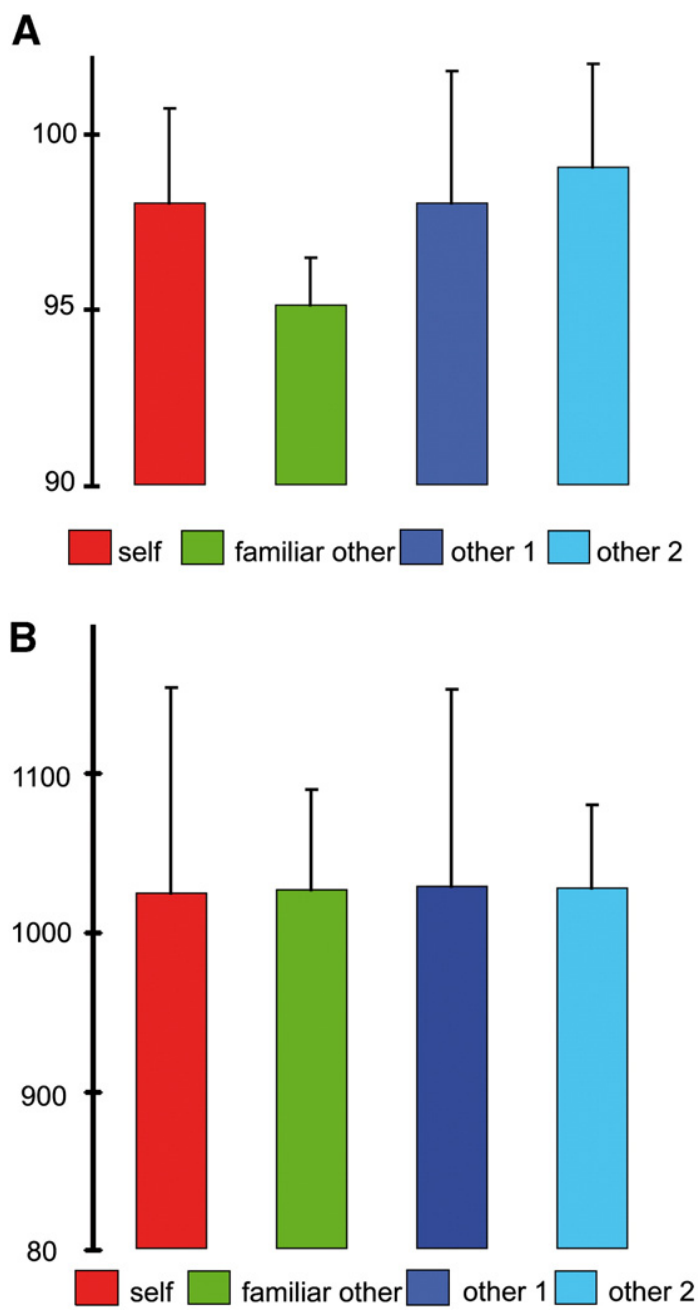

Fig. 1. (A) Accuracy of responses shown in percentage (\%) across conditions: self [red], familiar other [green], other 1 (other body contrasted to self; [dark blue]) and other 2 (other body contrasted to familiar other; [light blue]). Error bars represent standard error of the mean. (B) Means of the reaction times (RT) in ms across conditions: self, familiar other, other 1 and other 2. Error bars represent standard error of the mean. 'familiar other', RT =1030 ms, 96\% correct. The mean RTs and percentage correct did not differ significantly between categories (repeated measures ANOVA).

\section{Body-identification network}

The GLM RFX multi-subject analysis ( $t(9)>3, p<0.05$ uncorrected) did not reveal any specific brain region activated by the contrast [mirror >camera] or [camera > mirror] within 'self' or 'familiar other' category, therefore this factor was left out of further analyses. To rule out the possible effect of 'task set' at the brain level a RFX GLM whole brain analysis $(t(9)>3, p<0.05$ uncorrected) was performed for the contrasts ['others 1' (others from the 'self' task)> 'others 2' (others from the 'familiar other' task) and ['others 2'> 'others 1'], which did not yield any significant effect.

To investigate the cortical areas involved in body identification, we conducted a whole brain multi-subject GLM conjunction analysis for the contrasts [self $>$ other] and [familiar other $>$ other]. This should disclose all areas exhibiting responses to both contrasts of interest. The analysis revealed a network of the co-activated brain areas in the right fronto-parietal cortex. As explained above, we use the term 'network' to refer to the fact that more than two cortical regions functionally responded to a certain condition. We do not mean to imply anatomical or functional interconnectedness. BOLD signal modulation for the conjunction [self $>$ other and familiar other $>$ other] was observed in the inferior frontal gyrus bilaterally (IFG; Tal. $x=32$, $y=20, z=3$, and $x=-28, y=20, z=6)$, in the anterior and posterior bank of the medial frontal gyrus (MFG; Tal. $x=41, y=31, z=16$ and Tal. $x=50$, $y=7, z=26$ ), in the cingulate gyrus (CG; Tal. $x=3, y=14, z=38$ ), in the central and post-central sulci (CS; Tal. $x=38, y=-30, z=46$; PCS; Tal. $x=34, y=-52, z=39$ ), in the inferior parietal lobe (IPL; Tal. $x=27, y=-73$, $z=30$ ) and in the fusiform body area (FBA; Tal. $x=38, y=-46, z=-13$ ), all in the right hemisphere (Fig. 2).

Investigating the effect of body ownership we contrasted the activation induced by 'self and 'familiar other'. At the level of singlesubject analysis this contrast revealed a consistent activation in the right parietal lobe. The GLM RFX multi-subject analysis disclosed specific ROIs for the contrast [self $>$ familiar other] in the inferior parietal lobe (IPL Tal. $x=22, y=-71, z=35,468$ active voxels) and in the inferior parietal sulcus (IPS; Tal. $x=32, y=-71, z=10,834$ active voxels) of the right hemisphere, and further in the posterior orbital gyrus (pOrbG; Tal. $x=-32, y=30, z=$ $-5,398$ active voxels) and in the lateral occipital gyrus (LOG; Tal. $x=-44$, $y=-68, z=-15,158$ active voxels) in the left hemisphere (Fig. 3 ). This 'self' specific activation remained significant after correction for multiple comparisons; $t(9)>2.5 ; p<0.05$. The deconvolution time course graphs show the shape of the estimated BOLD response across conditions indicating that the main effects were not generated by noise or negative BOLD signal. (Fig. 3B).

To examine whether these 'self specific regions correspond to the regions activated in the body detection tasks we compared the respective maps. Unexpectedly, there was no overlap, suggesting different networks for the discrimination between self and familiar body (self-identification) and the processing of general body related information (body-detection).

\section{Mapping of the body selective areas}

To investigate whether body selective areas are modulated by identity or ownership we defined body selective regions of interest (ROIs) based on the mapping experiment, and subsequently tested whether these ROIs were modulated by the following contrasts [familiar other $>$ other], [self $>$ other] and [self $>$ familiar other] (Fig. 4).

We performed an explorative whole brain multi-subject RFX GLM analysis for the contrast [bodies $>$ objects] at $t(9)>3 ; p<0.05$ (corrected for multiple comparisons) and identified body selective ROIs in right EBA (Tal. $x=42, y=-63, z=10$ ), right FBA (Tal. $x=38, y=-65, z=-15$ ), left EBA 
A

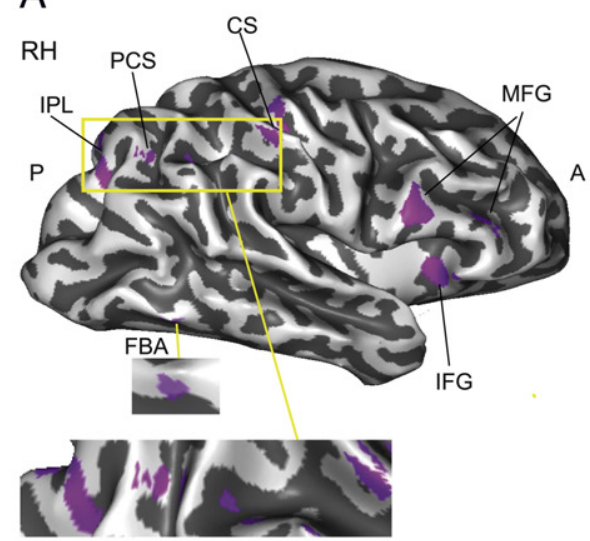

B

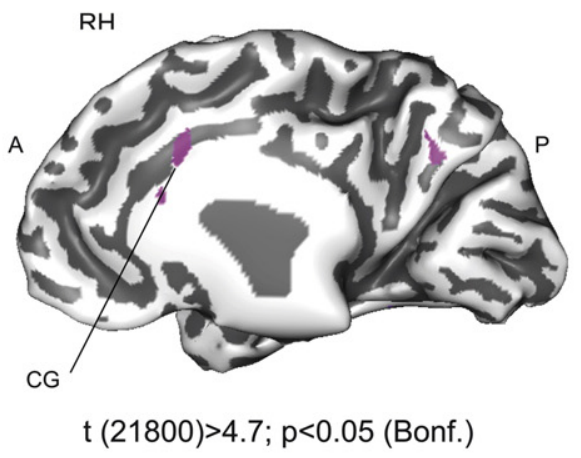

C

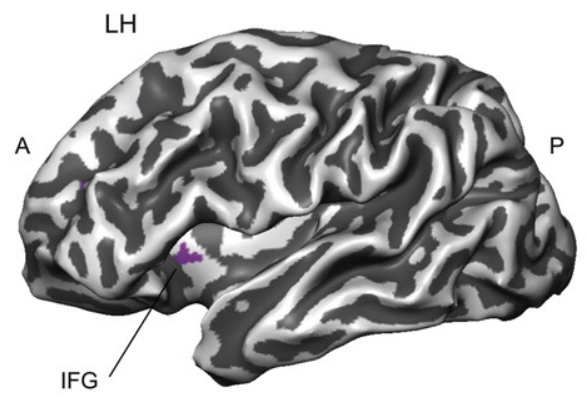

D
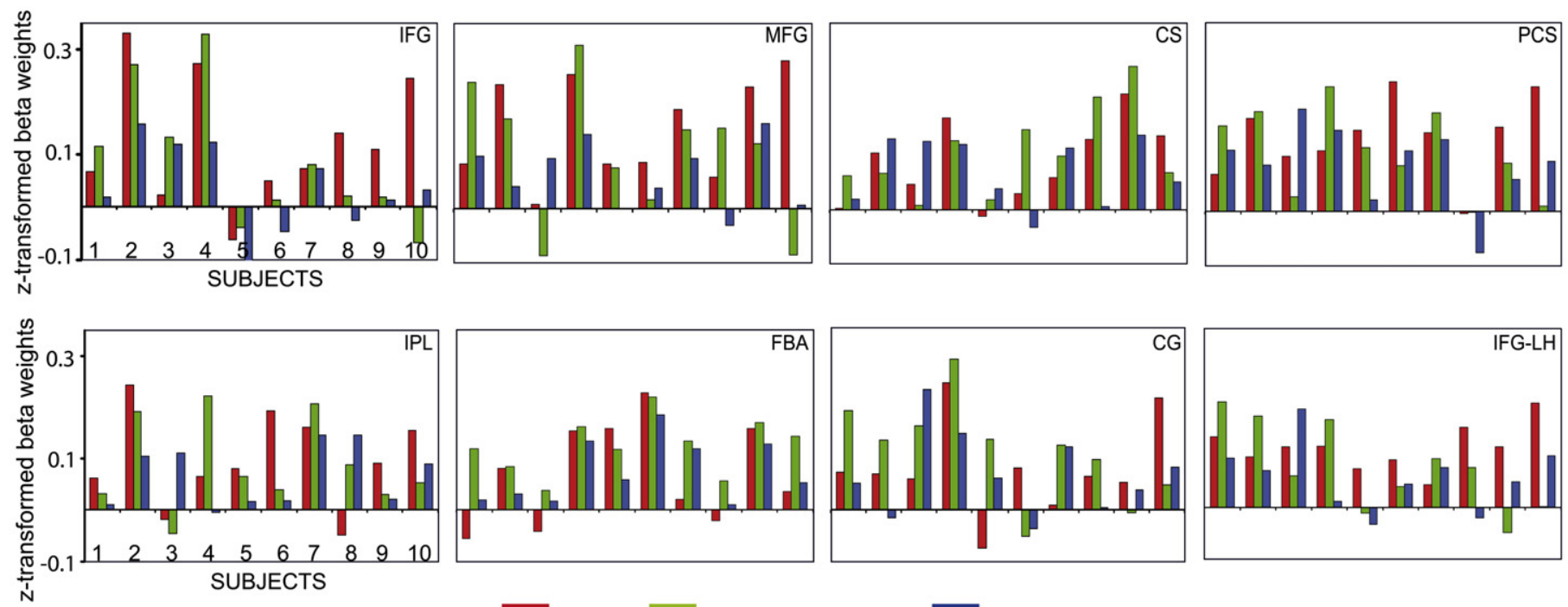

SELF

FAMILIAR OTHER

OTHERS

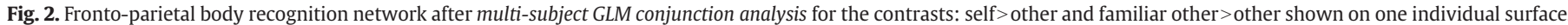

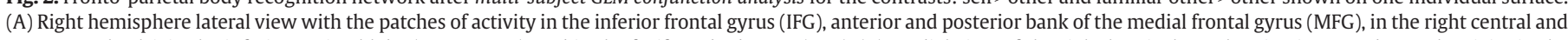

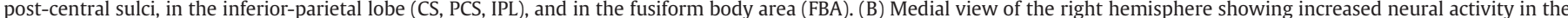

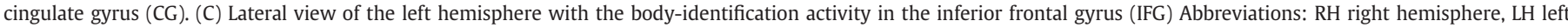

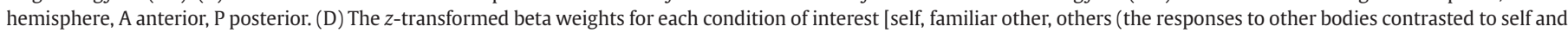

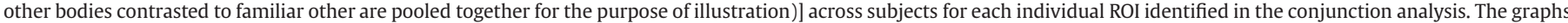
illustrate that the main conjunction effects were not generated by noise or negative BOLD accross subjects and ROIs.

(Tal. $x=-43, y=-73, z=-7)$ and in right inferior parietal lobe $\operatorname{IPL}(x=30$, $y=-52, z=33$ ).

At the level of single subject analyses right EBA ROI was identified in all 10 subjects, FBA in 8, right IPL in 9 and left EBA in 8 subjects [see Supplementary data; Table 1 (A-D)].

We then tested whether these body selective ROIs are modulated by the contrasts [familiar other $>$ other], [self $>$ other] and [selffamiliar other]. The right EBA activity was not significantly modulated with any of these contrasts even though a sporadic modulation was observed at the level of single-subjects [Supplementary data; Table 1 (A)]. Right FBA was significantly modulated with the contrasts [self $>$ other] and [familiar other $>$ other]. Left EBA was modulated by the contrast [self $>$ other]. Only the area in the right inferior parietal lobe showed a significant modulation with all contrasts of interest (Table 1).

In a further analysis, we found a significant interaction between condition and ROI in the activation patterns. This was due to a difference between the pattern of beta values in right FBA compared to all other body selective ROIs (left EBA, right EBA and right IPL) in response to familiar versus unfamiliar bodies. The rm ANOVA based on the sum of 5 beta weights around the peak of the deconvolution time course for each condition and individual subject demonstrated a significant interaction between condition and ROI $(F(6,54)=3.379$, $p=0.007$ ) (repeated measure ANOVA test) as well as a main effect of ROI $(F(3,27)=5.481, p=0.004)$. Planned comparisons (Helmert contrasts) revealed that the main effect of ROI was due to greater beta values in right FBA compared to all other ROIs $(F(1,9)=11.243$, $p=0.008$ ) as well as greater beta values in right IPL as compared to the right EBA $(F(1,9)=6.220, p=0.0342)$. The interaction between condition and ROI was due to a greater difference between 'self' compared to the average of 'familiar other' and 'other' in the right IPL $(F(1,9)=9.006, p=0.015)$ than in the right EBA, and a greater difference between 'familiar other' and 'other' in the right FBA compared to the average of this difference in all other ROIs $(F(1,9)=5.212, p=0.048)$.

\section{Discussion}

The goal of this study was to differentiate between cortical areas devoted to the analysis of body related information: 1 .) the processing of visual, body specific features (body-detection), 2.) the distinction 
A

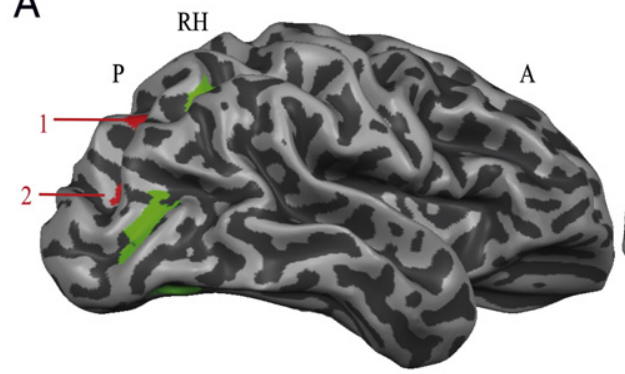

self $>$ familiar other; $\mathrm{t}(9)>2.5 ; \mathrm{p}<0.05$ (corrected)
B

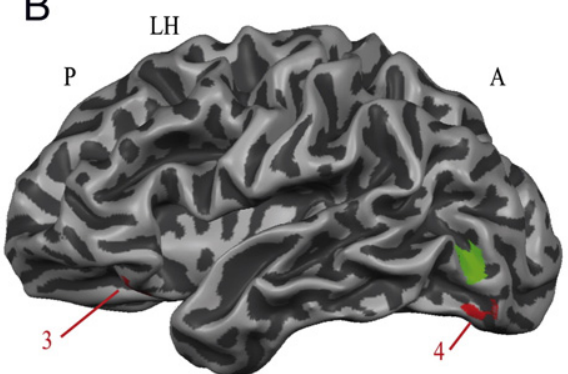

bodies $>$ objects; $\mathrm{t}(9)>2.5 ; \mathrm{p}<0.05$ (corrected)

$\mathrm{C}_{\mathrm{s}}$

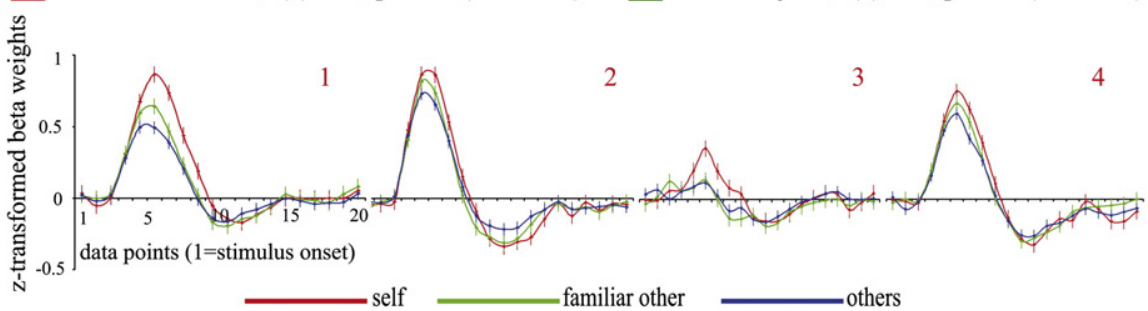

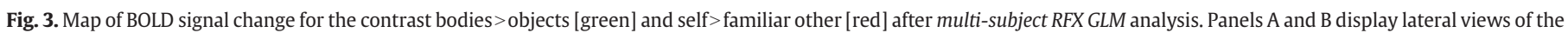

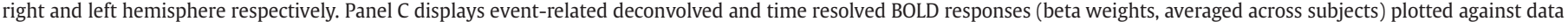

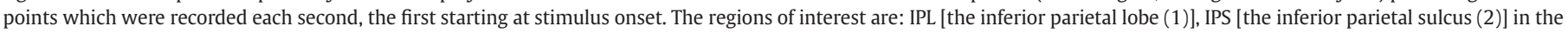

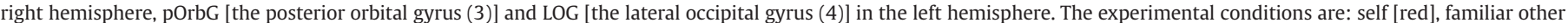

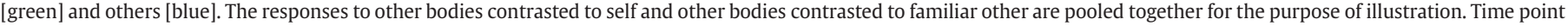

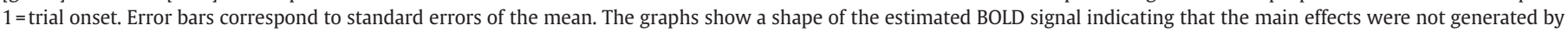
noise or negative BOLD. Abbreviations: RH right hemisphere, LH left hemisphere, A anterior, P posterior.

between familiar and unfamiliar bodies (body-identification) and 3.) the distinction of one's own body (self-identification).

\section{Behavioral results}

In the behavioral part of our research repeated measure ANOVA revealed that the accuracy of responses to the images of 'others' was significantly higher than to the images of 'familiar other', which might be due to the fact that the familiarization with the 'familiar other' image was either too brief or too close to the scanning. However, this effect was not shown in the RTs. The accuracy for 'self' was significantly higher than that for 'familiar other', reflecting the high degree of familiarity with one's own body and the limited time allowed for the familiarization with one of the other bodies. Finally, there was no significant difference in responses to mirror versus camera images, which suggests that subjects did not perform mental inversions for classifications.

\section{Body-detection network}

Our mapping results confirm the existence of brain areas specialized in the processing of visual features of bodies, the "body-
A

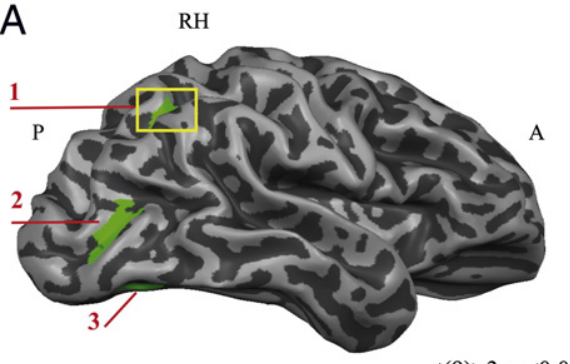

$\mathrm{t}(9)>3 ; \mathrm{p}<0.05$ (corrected)

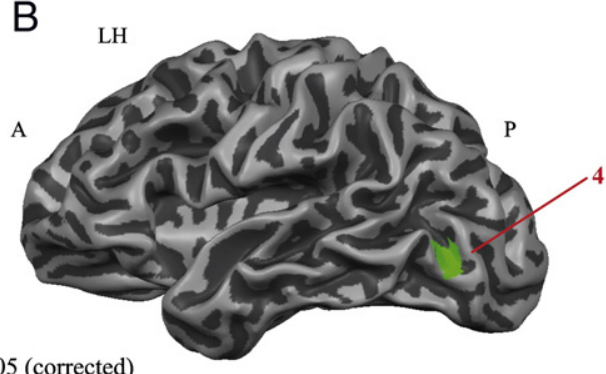

C

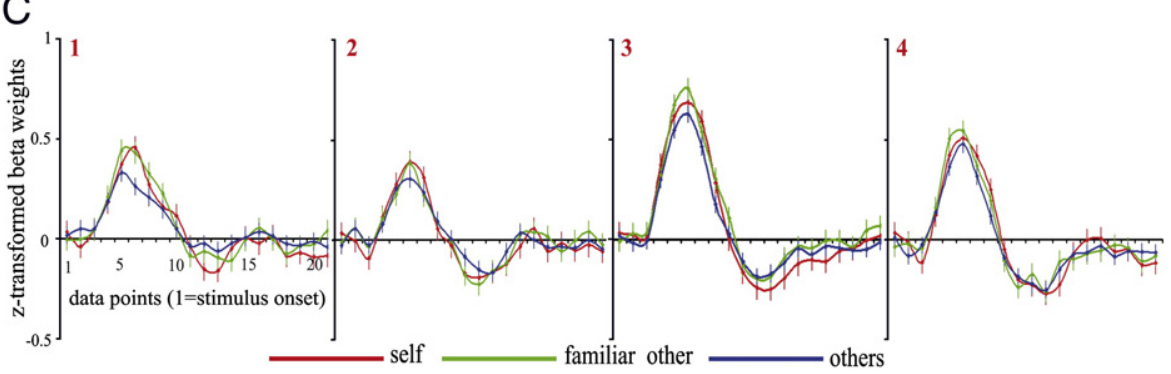

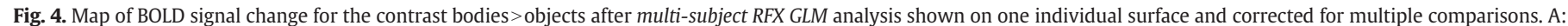

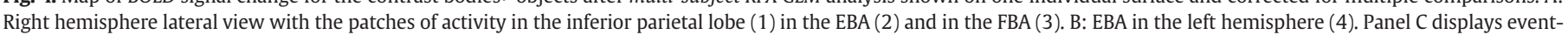

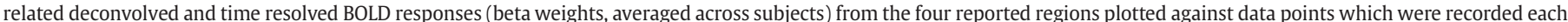

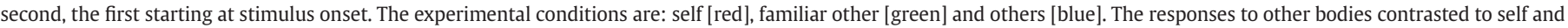

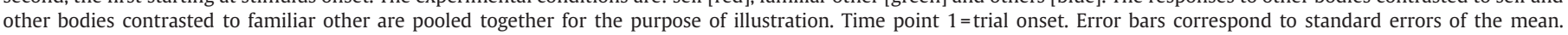
Abbreviations: $\mathrm{RH}$ right hemisphere, $\mathrm{LH}$ left hemisphere, $\mathrm{A}$ anterior, $\mathrm{P}$ posterior. 
detection network'. This network of functionally co-activated brain areas comprised the EBA, the FBA and the IPL in the right hemisphere and the EBA in the left hemisphere (Fig. 4). The network showed significant activation both in the group and in the single subject analyses [see Supplementary data; Table 1 (A-D)].

Thus, in addition to the well established EBA and FBA (Downing et al., 2001, Peelen and Downing 2005, Schwarzlose et al., 2005, Downing et al., 2006, Peelen et al., 2006) the present study disclosed a third body specific area in the right IPL that we have already identified in a previous study (Hodzic et al., 2008). This agrees with the notion that the parietal lobe plays an important role in the representation of body related information (Ehrsson et al., 2005, Pellijeff et al., 2006, Shimada et al., 2005). The IPL has been suggested to be involved in the processing of proprioceptive and visual signals relevant for body scheme representation (Jackson and Decety 2004, Uher et al., 2005) and in the feeling of the sense of agency of the observed limb movement (Chaminade and Decety 2002). In addition, lesions in the IPL have been shown to interfere with the ability to imitate gestures (Chaminade et al., 2005).

Electrophysiological recordings from a putative homologue of this area in non human primates revealed neurons monitoring limb positions (Sakata et al., 1973, Mountcastle et al., 1975, Graziano et al., 2000). The data are compatible with the present result showing that this parietal area is not only involved in the identification of bodies and their parts but also in the distinction of familiarity and ownership.

Taylor et al., (2007) suggested that the FBA is responsible for the integration of body parts into representations of whole bodies while EBA is confined to the analysis of body parts. Thus, the FBA might accomplish similar functions in body perception as the FFA in face perception (Loffler et al., 2005). In a recent review, Peelen and Downing (2007) proposed that the FBA might play a role in encoding not only bodies but also their identity. The present data provide evidence that FBA participates in assigning identities to the images of bodies and that the pattern of responses related to the body images clearly differ between FBA and EBA.

\section{Body-identification network}

Recognition of one's own face involves specific cortical areas that differ from the areas involved in general processing of faces (Platek et al., 2006, Sugiura et al., 2005, Uddin et al., 2005). Our data suggest a similar segregation for the processing of body related information. The network concerned with the identity of bodies (contrasts [familiar other $>$ other $]+[$ self $>$ other $]$ ) comprised in addition to the FBA also the areas in the right inferior parietal lobe and frontal cortical regions.

The FBA but not the EBA exhibited differential responses to familiar and unfamiliar bodies and the areas in the IPL responded differentially not only to familiar and unfamiliar bodies but distinguished also between 'self' and 'familiar other' (see Table 1 and the Supplementary data). In addition to the body specific areas, distinction between familiar and unfamiliar bodies involved further areas in the frontal lobe (IFG and MFG) (Fig. 2). The recognition of one's own body involved the IPL (inferior parietal lobe) and the IPS (inferior parietal sulcus) in the right hemisphere, and pOrbG (posterior orbital gyrus) and LOG (lateral occipital gyrus) in the left hemisphere (Fig. 3).

Comparison of the responses to self versus familiar other revealed self-specific responses in the IPL, IPS of the right hemisphere but not in the FBA. However, the FBA showed a clear response in the conjunction analysis for the contrasts [self $>$ other] and [familiar other $>$ other] (Fig. 2), indicating that FBA is involved in the encoding of the identity of bodies but not in the representation of self. EBA, in contrast, seems to be involved neither in the identification of individual bodies nor in the attribution of self or ownership. These findings differ from the data showing that EBA sorts body part images by identity (Myers and Sowden 2008). The dissimilarity in results might be due to different experimental paradigms (body parts vs. whole [headless] bodies). As our data show involvement of FBA in body identification, we propose that the initial processing of whole body image is performed in the EBA where bodies are processed at the level of parts. The next level of processing, the assignment of body identity, is performed in the FBA of the right hemisphere. These results are in line with the findings that EBA analyses bodies at the level of parts being the analog of the OFA (occipital face area) and that FBA processes bodies as the whole, being the analog of the FFA (fusiform face area) (Taylor et al., 2007).

The involvement of the IFG in body identification is in line with the findings about the role of frontal regions in face identification and self attribution of faces and voices (Nakamura et al., 2001, Platek et al., 2004, Platek et al., 2006, Sugiura et al., 2006, Sui and Han 2007, Uddin et al., 2005, Uddin et al., 2006). The association of body identification with frontal activation is also in agreement with the evidence that patients exhibiting a delusional misidentification syndrome (DMS) exhibit lesions in the right frontal lobe (Feinberg and Keenan, 2005).

The inferior parietal lobe (IPL) is another region where self specificity was observed. This region is involved in the processing of body related information and sense of agency (see above) and it also plays a role in the distinction between one's own face and that of familiar others (Platek et al., 2006, Uddin et al., 2005). A TMS study by Uddin et al., (2006) has indicated a causal relationship between this region and self-identification.

Although our data do not allow us to exclude attention-related effects as causes of the enhanced responses to the images of the subjects' own and familiar body, we believe that the enhancement is more specifically related to the activation of a network encoding identity and ownership. If attention would have been a major cause of the effects, we would have expected differential activations of networks involving further frontal cortices and/or limbic structures and we would have predicted more symmetric bilateral activation patterns. Yet another argument against an influence of attention on our data is the lack of identity-related BOLD signal modulation in the classical EBAs of the left and right hemisphere.

Furthermore, studying neural correlates of the assignment of the ownership to the seen body image we directly contrasted conditions from separate runs; namely self $>$ familiar other (Fig. 3), opening up the possibility of the influence of 'task set' or instruction on our data. Since cortical areas involved in the assignment of ownership were in close proximity to parietal "body" and "self-selective" regions found in a previous study without any instruction related to body recognition (Hodzic et al., 2008) we interpret our data as being mainly stimulus and not instruction driven. Independent of the exact contribution of top-down and bottom-up influences in IPL and IPS, we propose that the activated areas are possible substrates of the 'abstract concept of bodily self.

In sum, our data indicate segregation between fronto-parietal bodyidentification network and the parieto-occipital body-detection network, suggesting that processing of body images involves several subprocesses: 1 . the encoding of body parts (EBA), 2. the integration of body parts into whole bodies and the identification of individual bodies (FBA), 3. the identification of one's own body (IPL) and finally, 4. the activation of a fronto-parietal network that encodes in a more abstract way the concept of self, which is activated not only by body related cues.

At the behavioral level we did not find significant differences in the reaction times or accuracy of responses when the viewpoints or perspectives of the body images are manipulated (mirror vs. camera images). Similarly, at the cortical level, the present event related approach confirmed that the BOLD signal in EBA is not influenced by manipulations of perspective, identity or ownership of the body related cues confirming our previous findings (Hodzic et al., 2008), as well as those of other authors (Chan et al., 2004, Saxe et al., 2006a,b). However, this is at variance with claims that EBA might be crucial for the assessment of body identity (Urgesi et al., 2007b) and recent findings that the EBA actively discriminates body parts' identity (Myers and Sowden 2008). 
The areas identified in this study have been activated by images of static, headless bodies in upright position. Thus, further subsystems are likely to be involved when movement and posture are provided as additional cues for detection and identification of bodies.

\section{Acknowledgment}

We are grateful to $\mathrm{S}$. Weigelt for contributions to experimental design and data analysis.

\section{Appendix A. Supplementary data}

Supplementary data associated with this article can be found, in the online version, at doi:10.1016/j.neuroimage.2009.01.027.

\section{References}

Chaminade, T., Decety, J., 2002. Leader or follower? Involvement of the inferior parieta lobule in agency. Neuroreport 28 (13), 1975-1978.

Chaminade, T., Meltzoff, A.N., Decety, J., 2005. An fMRI study of imitation: action representation and body scheme. Neuropsychologia 43, 115-127.

Chan, A.W., Peelen, M.V., Downing, P.E., 2004. The effect of viewpoint on body representation in the extrastriate body area. Neuroreport 25, 2407-2410.

Cunnington, R., Windischberger, C., Robinson, S., Moser, E., 2006. The selection of intended actions and the observation of others' actions: a time-resolved fMRI study. Neuroimage 29, 1294-1302.

Dale, A.M., Buckner, R.L., 1997. Selective averaging of rapidly presented individual trials using fMRI. Hum Brain Mapping 5, 329-340.

DeSimone, R., Albright, T.D., Gross, C.G., Bruce, C., 1984. Stimulus-selective properties of inferior temporal neurons in the macaque. J. Neurosci. 4, 2051-2062.

Downing, P.E., Jiang, Y., Shuman, M., Kanwisher, N., 2001. A cortical area selective for visual processing of the human body. Science 293, 2470-2473.

Downing, P.E., Chan, A.W.Y., Peelen, M.V., Dodds, C.M., Kanwisher, N., 2006. Domain specificity in visual cortex. Cereb. cortex 16, 1453-1461.

Ehrsson, H.H., Kito, T., Sadato, N., Passingham, R.E., Naito, E., 2005. Neural substrate of body size: Illusory feeling of shrinking of the waist. PLoS Biol 3, e412.

Feinberg, T.E., Keenan, J.P., 2005. Where in the brain is the self? Conscious. Cogn. 14 661-678.

Forman, S.D., Cohen, J.D., Fitzgerald, M., Eddy, W.F., Mintun, M.A., Noll, D.C., 1995 Improved assessment of significant activation in functional magnetic-resonanceimaging (fMRI) - Use of a cluster-size threshold. Magn Reson Med 33, 636-647.

Friston, K.J., Rotshtein, P., Geng, J.J., Sterzer, P., Henson, R.N., 2006. A critique of functional localisers. Neuroimage 30 (4), 1077-1087.

Gauthier, I., Tarr, M.J., Anderson, A.W., Skudlarski, P., Gore, J.C., 1999. Activation of the middle fusiform 'face area' increases with expertise in recognizing novel objects. Nat. Neurosci. 2 (6), 568-573.

Graziano, M.S., Cooke, D.F., Taylor, C.S., 2000. Coding the location of the arm by sight Science 290, 1782-1786.

Grezes, J., Frith, C.D., Passingham, R.E., 2004. Inferring false beliefs from the actions of oneself and others: An fMRI study. Neuroimage 21, 744-750.

Gross, C.G., Rocha-Miranda, C.E., Bender, D.B., 1972. Visual properties of neurons in inferotemporal cortex of the Macaque. J. Neurophysiol. 35, 96-111.

Haxby, J.V., Gobbini, M.I., Furey, M.L., Ishai, A., Schouten, J.L., Pietrini, P., 2001. Distributed and overlapping representations of faces and objects in ventral temporal cortex. Science 293, 2425-2430.

Hodzic, A., Muckli, L., Singer, W., Stirn, A., 2008. Cortical responses to self and others. Human Brain Mapping (doi:10.1002/hbm.20558).

Ishai, A., Ungerleider, L.G., Martin, A., Haxby, J.V., 2000. The representation of objects in the human occipital and temporal cortex. J. Cog. Neurosci. 2, 35-51.

Jackson, P.L., Decety, J., 2004. Motor cognition: a new paradigm to study self-other interactions. Curr. Opin. Neurobiol. 14, 259-263.

Kanwisher, N., McDermott, J., Chun, M.M., 1997. The fusiform face area: a module in human extrastriate cortex specialized for face perception. J. Neurosci. 17, 4302-4311.

Kiani, R., Esteky, H., Mirpour, K., Tanaka, K., 2007. Object category structure in response patterns of neuronal population in monkey inferior temporal cortex. J. Neurophysiol. 97, 4296-4309.

Kovacs, G., Zimmer, M., Banko, E., Harza, I., Antal, A., Vodnyanszky, Z., 2005. Electrophysiological correlates of visual adaptation to faces and body parts in humans. Cerebral Cortex 16, 742-753.

Loffler, G., Yourganov, G., Wilkinson, F., Wilson, H.R., 2005. fMRI evidence for the neural representation of faces. Nat. Neurosci. 8, 1386-1390.

Mountcastle, V.B., Lynch, J.C., Georgopoulos, A., Sakata, H., Acuna, C., 1975. Posterior parietal association cortex of the monkey: command functions for operations within extrapersonal space. J. Neurophysiol. 38, 871-908.

Myers, A., Sowden, P.T., 2008. Your hand or mine? The extrastriate body area. doi: 10.1016/j.neuroimage.2008.05.045

Nakamura, K., Kawashima, R., Sugiura, M., Kato, T., Nakamura, A., Hatano, K., Naguma, S. Kubota, K., Fukuda, H., Ito, K., Kojima, S., 2001. Neural substrates for recognition of familiar voices: a PET study. Neuropsychologia 39, 1047-1054.
O'Toole, A.J., Jiang, F., Abdi, H., Haxby, J.V., 2005. Partially distributed representations of objects and faces in ventral temporal cortex. J. Cog. Neurosci. 17 (4), 580-590.

Peelen, M.V., Downing, P.E., 2005. Selectivity for the human body in the fusiform gyrus. J. Neurophysiol. 63, 603-605.

Peelen, M.V., Downing, P.E., 2007. The neural basis of visual body perception. Nat. Rev. Neuroscience 8, 636-648.

Peelen, M.V., Wiggett, A.J., Downing, P.E., 2006. Patterns of fMRI activity dissociate overlapping functional brain areas that respond to biological motion. Neuron 16 , $815-822$

Pellijeff, A., Bonilha, L., Morgan, P.S., McKenzie, K., Jackson, S.R., 2006. Parietal updating of limb posture: an event-related fMRI study. Neuropsychologia 44, 2685-2690.

Pinsk, M.A., DeSimone, K., Moore, T., Gross, C.G., Kastner, S., 2005. Representations of faces and body parts in macaque temporal cortex: a functional MRI study. Proc. Natl. Acad. Sci. U. S. A. 102, 6996-7001.

Platek, S.M., Keenan, J.P., Gallup Jr., G.G., Mohamed, F.B., 2004. Where am I? The neurological correlates of self and other. Brain Res. 19, 114-122.

Platek, S.M., Loughead, J.W., Gur, R.C., Busch, S., Ruparel, K., Phend, N., Panyavin, I.S., Langleben, D.D., 2006. Neural substrates for functionally discriminating self-face from personally familiar faces. Hum. Brain Mapp. 27, 91-98.

Pourtois, G., Peelen, M.V., Spinelli, L., Seeck, M., Vuilleumier, P., 2007. Direct intracranial recording of body-selective responses in human extrastriate visual cortex. Neuropsychologia 45, 2621-2625.

Puce, A., Allison, T., Asfgari, M., Gore, J.C., Mccarthy, G., 1996. Differential sensitivity of human visual cortex to faces, letterstrings, and textures: a functional magnetic resonanace imaging studies. J. Neurosci. 16, 5205-5212.

Ramnani, N., Miall, R.C., 2004. A system in the human brain for predicting the actions of others. Nat. Neurosci. 7, 5-6.

Sakata, H., Takaoka, Y., Kawarasaki, A., Shibutani, H., 1973. Somatosensory properties of neurons in the superior parietal cortex (area 5) of the rhesus monkey. Brain Res 21, 85-102.

Saxe, R., Brett, M., Kanwisher, N., 2006a. Divide and conquer: a defense of functional localizers. Neuroimage 30 (4), 1088-1096.

Saxe, R., Jamal, N., Powell, L., 2006b. My body or yours? The effect of visual perspective on cortical body representations. Cereb. Cortex 16, 178-182.

Schwarzlose, R.F., Baker, C.I., Kanwisher, N., 2005. Separate face and body selectivity in the fusiform gyrus. J. Neurosci. 23, 11055-11059.

Shimada, S., Hiraki, K., Oda, I., 2005. The parietal role in the sense of self-ownership with temporal discrepancy between visual and proprioceptive feedbacks. Neuroimage $15,1225-1232$.

Sugiura, M., Watanabe, J., Maeda, Y., Matsue, Y., Fukuda, H., Kawashima, R., 2005. Cortical mechanisms of visual self-perception. Neuroimage 24, 143-149.

Sugiura, M., Sassa, Y., Jeong, H., Miura, N., Akitsuki, Y., Horie, K., Sato, S., Kawashima, R., 2006. Multiple brain networks for visual self-recognition with different sensitivity for motion and body part. Neuroimage 32, 1905-1917.

Sui, J., Han, S., 2007. Self-construal priming modulates neural substrates of selfawareness. Psychological Science 18, 861-866.

Talairach, J., Tournoux, P., 1988. Co-Planar Stereotactic Atlas of the Human Brain. Thieme, Stuttgart/New York

Taylor, J.C., Wiggett, A.J., Downing, P., 2007. Functional MRI analysis of body and body part representations in the extrastriate and fusiform body areas. J. Neurophysiol. 98, $1626-1633$.

Thierry, G., Pegna, A.J., Dodds, C., Roberts, M., Basan, S., Downing, P., 2006. An eventrelated potential component sensitive to images of the human body. Neuroimage $32,871-879$.

Tsao, D.Y., Freiwald, W.A., Knutsen, T.A., Mandeville, J.B., Tootell, R.B., 2003. Faces and objects in macaque cerebral cortex. Nat. Neurosci. 6, 989-995.

Tsao, D.Y., Freiwald, W.A., Tootell, R.B., Livingstone, M.S., 2006. A cortical region consisting entirely of face-selective cells. Science 3, 670-674.

Uddin, L.Q., Kaplan, J.T., Molnar-Szakacs, I., Zaidel, E., Iacoboni, M., 2005. Self-face recognition activates a frontoparietal mirror network in the right hemisphere: an event-related fMRI study. NeuroImage 25, 926-935.

Uddin, L.Q., Molnar-Szakacs, I., Zaidel, E., Iacoboni, M., 2006. rTMS to the right inferior parietal lobule disrupts self-other discrimination. Soc. Cog. Affect Neurosci. 1, $65-71$

Uher, R., Murphy, T., Friederich, H.C., Dalgleish, T., Brammer, M.J., Giampietro, V., Phillips, M.L., Andrew, C.M., Williams, S.C., Campbell, I.C., Treasure, J., 2005. Functional neuroanatomy of body shape perception in healthy and eating-disordered women. Biol. Psychiatry 15, 990-997.

Urgesi, C., Berlucchi, G., Aglioti, S.M., 2004. Magnetic stimulation of extrastriate body area impairs visual processing of nonfacial body parts. Curr. Biol. 14, 2130-2134.

Urgesi, C., Calvo-Merino, B., Haggard, P., Aglioti, S.M., 2007a. Transcranial magnetic stimulation reveals two cortical pathways for visual body processing. J. Neurosci. $25,8023-8030$

Urgesi, C., Candidi, M., Ionta, S., Aglioti, S.M., 2007b. Representation of body identity and body actions in extrastriate body area and ventral premotor cortex. Nat. Neurosci. $10,30-31$.

Vogeley, K., Bussfeld, P., Newen, A., Herrmann, S., Happe, F., Falkai, P., Maier, W., Shah, N.J., Fink, G.R., Zilles, K., 2001. Mind reading: Neural mechanisms of theory of mind and self-perspective. Neuroimage 14, 170-181.

Wachsmuth, E., Oram, M.W., Perrett, D.I., 1994. Recognition of objects and their component parts: responses of single units in the temporal cortex of the macaque. Cereb. Cortex 4, 509-522.

Zaitsev, M., Henning, J., Speck, O., 2004. Point spread function mapping with parallel imaging techniques and high acceleration factors: fast, robust, and flexible method for echo-planar imaging distortion correction. Magn. Reson. Med. 52, 1156-1166. 